\title{
La nueva gerencia pública como limitante de la mediación transformativa en México
}

The new public management as limiting of transformative mediation in Mexico

A nova gestão pública como limitação da mediação transformadora no México

José Antonio Serrano Morán

Universidad Autónoma de Nayarit, México serranomoran@gmail.com https://orcid.org/0000-0002-2669-6902

Nicté Rosas Topete Universidad Autónoma de Nayarit, México nicte.uan@gmail.com https://orcid.org/0000-0002-6009-1835

Edgar Gabriel Ávila Verdín Universidad Autónoma de Nayarit, México edgaravilav1@hotmail.com https://orcid.org/0000-0001-6004-0029 


\section{Resumen}

El objetivo del presente artículo fue analizar la figura sobre la nueva gerencia pública y su impacto en el modelo transformativo de la mediación. Para ello, se usaron los métodos deductivo, documental, discursivo, sistemático jurídico y funcionalista. Los resultados señalan que el eje denominado rendición de cuentas — al focalizar su estudio en términos estadísticos cuantitativos, mas no cualitativos - obliga a los centros de mediación en sede judicial a priorizar la generación de estadísticas sobre acuerdos firmados por encima de la transformación de la conducta de los mediados, lo que impacta de forma negativa en el modelo transformativo y en la no reincidencia en los conflictos.

La limitación más importante fue la obtención de datos estadísticos específicos sobre el número de asuntos presentados y acuerdos signados ante los centros de mediación, siendo el principal hallazgo del estudio, en el sentido de que el Estado mexicano utiliza el modelo clásico de medición, el cual se centra en la obtención de un acuerdo entre las partes, con lo que se sostiene una política de reacción que se aleja cada vez más de la política de prevención del conflicto. Esta situación sirve para concluir que se debe eliminar la política de la nueva gerencia pública en los centros de mediación de sede judicial para brindarles la oportunidad de utilizar el método transformativo para la resolución de conflictos.

El supuesto hipotético del presente artículo consistió en fundamentar la necesidad de aplicar el modelo transformativo para la mediación dentro del Poder Judicial en México; sin embargo, la ideología de la nueva gerencia pública que ha implementado el Estado mexicano dificulta en gran medida los beneficios y alcances de prevención de conflictos y no repetición de los hechos, por lo que es necesario cesar el funcionamiento de la mediación con base en los principios de la nueva gerencia pública para que se puedan adoptar los fines de la mediación transformativa.

Palabras clave: administración pública, mediación, resolución de conflictos, sistemas jurídicos. 


\section{Revista Iberoamericana \\ de las Ciencias Sociales y \\ Humanísticas}

ISSN: $2395-7972$

\section{Abstract}

The objective of this article was to analyze the figure on the new public management and its impact on the transformative model of mediation. For it, the deductive, documentary, discursive, legal systematic and functionalist methods were used. The results indicate that the axis called accountability — by focusing its study in quantitative statistical terms, but not qualitative - forces mediation centers in judicial headquarters to prioritize the generation of statistics on signed agreements over the transformation of behavior of the part mediated, which has a negative impact on the transformative model and the non-recidivism in conflicts.

The most important limitation was the obtaining of specific statistical data on the number of cases presented and agreements signed before the mediation centers, the main finding of the study being, in the sense that the Mexican State uses the classic mediation model, which it focuses on obtaining an agreement between the parts, thereby sustaining a policy of reaction that is increasingly moving away from the policy of conflict prevention. This situation serves to conclude that the policy of the new public management in the mediation centers of judicial headquarters should be eliminated to give them the opportunity to use the transformative method for conflict resolution.

The hypothetical assumption of this article consisted in substantiate the need to apply the transformative model for mediation within the judiciary in Mexico; However, the ideology of the New public management that the Mexican State has implemented greatly hinders the benefits and scope of conflict prevention and non-repetition of the facts, so it is necessary to cease the operation of mediation based on the principles of the new public management so that the aims of transformative mediation can be adopted.

Keywords: public administration, mediation, conflict resolution, legal systems.

\section{Resumo}

O objetivo deste artigo foi analisar a figura da nova gestão pública e seu impacto no modelo transformador de mediação. Para tanto, foram utilizados os métodos dedutivo, documental, discursivo, sistemático jurídico e funcionalista. Os resultados indicam que o eixo denominado accountability - por focar seu estudo em termos estatísticos quantitativos, mas não qualitativos - obriga os centros de mediação nas sedes judiciais a priorizar a geração de estatísticas sobre acordos firmados em detrimento da transformação de comportamento do 


\section{Revista Iberoamericana de las Ciencias Sociales y Humanísticas}

ISSN: $2395-7972$

meio, o que impacta negativamente o modelo transformador e a não reincidência em conflitos.

A limitação mais importante foi a obtenção de dados estatísticos específicos sobre o número de casos apresentados e acordos firmados junto aos centros de mediação, sendo o principal achado do estudo, no sentido de que o Estado mexicano utiliza o modelo clássico de mensuração, que centra-se na obtenção de um acordo entre as partes, sustentando assim uma política de reação que se distancia cada vez mais da política de prevenção de conflitos. Esta situação permite concluir que a política da nova gestão pública nos centros de mediação das sedes judiciais deve ser eliminada para lhes dar a oportunidade de utilizar o método transformador de resolução de conflitos.

O pressuposto hipotético deste artigo consistiu em fundamentar a necessidade de aplicar o modelo transformador de mediação no Poder Judiciário mexicano; No entanto, a ideologia da nova gestão pública que o Estado mexicano tem implementado dificulta sobremaneira os benefícios e alcance da prevenção de conflitos e da não repetição dos eventos, por isso é necessário cessar a operação de mediação com base no princípios da nova gestão pública para que os objetivos da mediação transformadora possam ser adotados.

Palavras-chave: administração pública, mediação, resolução de conflitos, sistemas jurídicos.

Fecha Recepción: Diciembre 2019

Fecha Aceptación: Julio 2020

\section{Introducción}

La simple aplicación de la mediación a casos concretos de forma discrecional — sin sentido del orden, ni objetivo fijo, ni modelo específico - es tan ocioso como un juicio sin pruebas. Es inocente creer que la mediación es un proceso de resolución de conflicto que se puede estandarizar para cualquier problema, pues ese pensamiento condenó al sistema jurisdiccional formal y sus sentencias por línea de producción, sin tomar en cuenta la individualización de las partes. Por eso, se deben establecer los modelos más idóneos dependiendo del conflicto que se pretenda solucionar en relación con los objetivos que las partes intentan lograr del proceso de mediación. Para ello, los modelos más ambiciosos son el transformativo y el asociativo, ya que prometen el cambio estructural de las personas, lo que en el modelo de mediación en sede judicial no es un tema menor y en diversas entidades federativas se considera irreal. 


\section{Revista Iberoamericana de las Ciencias Sociales y Humanísticas}

A efectos de estar en condiciones de estudiar y entender de mejor forma el fenómeno de la nueva gerencia pública (NGP), es necesario indagar en la evolución que ha tenido la administración pública en el mundo. En Alemania, donde tiene su cuna la tradición administrativa europea a través del concepto cameralismo, se asistió desde el siglo XVIII al desarrollo de una ciencia aplicada de la administración pública orientada a estudiar los principios de una administración eficaz y abordada principalmente desde dos perspectivas y momentos históricos diferentes (Gómez Días de León, 2015).

En Francia durante el siglo XVIII surgió la ciencia de la policía, que considera a la administración como el arte de dirigir los asuntos públicos y tiene un propósito esencialmente utilitario, teoría utilitarista que objetiviza todo lo que entra en su filosofía, inclusive a las personas (Gómez Díaz de Leon, 2015).

Finalmente, en Estados Unidos se pueden dividir en tres periodos las corrientes doctrinarias que dieron surgimiento a la administración pública: el periodo clásico, el periodo político y el periodo de "programas”. El clásico señala que la administración pública tiene como propósito esencial la racionalización de los métodos de actuación del Ejecutivo, y que es necesario erradicar de las prácticas de los servidores públicos el desorden empírico que dominaba la administración de ese entonces (Gómez Díaz de Leon, 2015).

Es así como se encuentra el origen de la gerencia pública a finales de los años 80 e inicios de los 90 en la escuela económica neoclásica de la escuela austriaca y de la opción pública estadounidense (Guerrero, 2003), basándose en algunos principios de la administración científica de Taylor y la administración burocrática del modelo de dominación legal-racional de Weber, dirigidos a desarrollar los factores intraorganizacionales de operación y que inciden en la modernización administrativa (León Corona y Cruz Badillo, 2014).

La nueva gerencia pública surge en el mundo anglosajón en buena medida para dar cuenta y superar las limitaciones del viejo modelo de desempeño gubernamental, estudiado en las primeras décadas del siglo pasado por Max Weber, razón por la cual al modelo de gobierno que él estudió se le conoce como el paradigma weberiano (Pineda Ortega, 2015).

Las ideas principales de este nuevo sistema se centran en intentar acercar la producción pública a los procedimientos de producción privada, pero sin trasladar directamente sus métodos — como propone el gerencialismo-, sino adecuando estos métodos a la producción pública dadas las peculiaridades que plantea (Cejudo, 2011). 


\section{Revista Iberoamericana \\ de las Ciencias Sociales y Humanísticas}

ISSN: $2395-7972$

El fenómeno de la nueva gerencia publica tampoco es nuevo en Latinoamérica; por ejemplo, en Colombia la NGP se puede encontrar desde 1992, con un marco normativo devenido de la Ley 30 de 1992 y del Decreto 1767 de 2006, a través del Sistema Nacional de Información de la Educación Superior (Salcedo Casallas, 2015).

En México, el tema de la administración pública tiene auge hasta que es contemplada como carrera universitaria en los años 70 , siendo las temáticas a tratar la burocracia, la organización y funcionamiento de la administración pública, la organización administrativa, la administración financiera, la reforma administrativa, la miscelánea y la ciencia de la administración (Gómez Días de León, 2015).

La crisis de eficacia, eficiencia y legitimidad —crónica especialmente en países de América Latina - obligó a reconsiderar el papel del gobierno, la exigencia de calidad en los servicios públicos, y de un mejor trato a los ciudadanos y usuarios de dichos servicios, lo que situó en un gran dilema al modelo administrativo clásico (Villareal Solis, 2015).

En los años 80, se desarrolló con cierta fuerza doctrinal una corriente de análisis de la administración bajo una perspectiva fundamentalmente economicista como consecuencia de los diversos economistas formados en Estados Unidos, que después se incrustaron en las filas de las instituciones públicas federales, principalmente en el Banco de México y en la Secretaría de Hacienda y Crédito Público. Esto -en conjunto con circunstancias presupuestarias restrictivas a raíz de las recurrentes crisis económicas en el país y asociado a un acelerado proceso de globalización - dio como resultado la transformación de la visión de la disciplina público-administrativa desde un enfoque tradicional hacia el que más tarde se constituiría como gestión pública, más específicamente la nueva gestión pública (Gómez Días de León, 2015).

La mediación como herramienta pacificadora y reparadora de relaciones interpersonales cuenta con diversos modelos que determinan objetivos y prioridades distintas dentro de un proceso mediador, contando con tres modelos consolidados hasta el momento: el modelo de Harvard, modelo transformativo y el modelo circular narrativo, de los cuales se desprenden más de doce diferentes subtipos, que cuentan con pequeñas diferencias en relación con los anteriores, o bien son el resultado de la combinación de dos o inclusive de los tres modelos señalados (Nató, 2006). 


\section{Revista Iberoamericana de las Ciencias Sociales y Humanísticas}

En 1994, año recordado por muchos eventos como el inicio de la rebelión social en Chiapas (México) o el sangriento enfrentamiento intertribal en Ruanda protagonizado por las etnias de los hutus y los tutsis, que tuvo como consecuencia un saldo de cerca de 800000 víctimas mortales y más de 2000000 de refugiados. En ese mismo año, Bush y Folger publicaron un texto que abrió - en materia de mediación — una nueva visión acerca de cómo afrontar el conflicto a través del fortalecimiento propio y el reconocimiento de los otros (Hernández Ramos, 2014). Asimismo, iniciaron una metodología que se centraría en la mejora o transformación de las relaciones humanas, y no tanto en la satisfacción de una determinada necesidad mediante el establecimiento de un acuerdo (Giménez Romero, 2001).

Específicamente, la mediación transformativa ayudó a atender tres importantes preocupaciones que habían emergido acerca de la dirección y evolución del ejercicio de la mediación en Estados Unidos y otras partes:

a) ¿Ofrece realmente la práctica de la mediación un proceso alternativo de intervención en conflictos?

b) ¿Ejercen los mediadores con un claro propósito?

c) ¿Puede la mediación preservar su capacidad propia como espacio no adjudicador de intervención en conflictos? (Folger, 2006).

Así pues, el aparato judicial está desfasado en relación con las necesidades reales de la sociedad y con su contexto económico y político, por lo cual es incapaz de dar una respuesta adecuada a las incesantes peticiones de administración de justicia que los ciudadanos reclaman día a día. Aunado a lo anterior, pareciera ser que en el sistema jurisdiccional estamos más preocupados por cuidar el formalismo y los rituales sagrados con los que se desarrolla un proceso que en dar debida solución a los intereses verdaderos que persiguen las partes afectadas y partícipes del conflicto. Esto trae como consecuencia una justicia burocratizada y poco efectiva, ajena a las necesidades de justicia de la ciudadanía y lejos de tener al menos una idea de cómo solucionarlas (Eiras Nordenstahl, 2010), aunque todo pareciera indicar que tampoco les urge el descubrirlo.

Como ya se ha mencionado, en el sistema de justicia actual, las víctimas toman un papel secundario, y son llamadas ante la autoridad solo para repetir las veces que sean necesarias los hechos de los cuales fueron víctima con el objetivo de describir lo sucedido y y para tartar de buscar detalles cada vez más ofensivos y destructivos en contra del presunto responsable. Sin embargo, la autoridad no percibe que esa constante repetición sistemática 


\section{Revista Iberoamericana de las Ciencias Sociales y Humanísticas}

ISSN: 2395 - 7972

de los hechos - escudriñando o construyendo un lado oscuro de los hechos - también lesiona a la víctima, quien es doblemente victimizada por el sistema en el que es forzada a funcionar si quiere "obtener justicia". En otras palabras, de lado ha quedado su verdadera intención, su sentir y su pensar, pues todo es sustituido por normas frías, formalismos inertes y pretensiones propias de un servidor público (Revilla González, 2007).

Por eso, Pacheco Pulido (2012) hace referencia a cinco aspectos que influyen en la situación actual del sistema penal: el incremento de la criminalidad, la inadecuada investigación del delito y los delincuentes, la deficiente integración de la carpeta de investigación, la sobrecarga de los tribunales, y la falta de satisfacción de los intereses y necesidades de las partes.

De esta manera, existe una deficiencia en el sistema penal con la función reeducativa o resocializante que se le encomendó, ya que en ningún momento se desprende de la realidad social en aquellas personas que han transitado por un proceso jurisdiccional formal, y que a la postre son sentenciados a compurgar una pena privativa de libertad en algún centro de reclusión sin que hayan desarrollado un cambio radical en su actuar cotidiano, en su estructura mental o en su forma de convivir y desarrollarse con el resto de la comunidad que lo rodea. Además, no se consideran las condiciones deplorables en las cuales se encuentran algunos centros de reinserción social —a nivel local y federal—, donde no se cuentan con los programas e infraestructuras necesarias para aproximarse a una verdadera resocialización y readaptación social. En otras palabras, si la finalidad del proceso penal es solamente mostrar la verdad histórica de los hechos para en consecuencia llegar a la verdad jurídica, en qué momento el proceso penal pretende resocializar o reeducar al delincuente si se ignoran las relaciones interpersonales de los protagonistas del delito, así como sus necesidades personales (Comisión Nacional de los Derechos Humanos [CNDH], 2018)

De igual manera, la recomendación R (87) 21 adoptada en septiembre de 1987 sobre asistencia a las víctimas y prevención de la victimización del comité de ministros del Consejo de Europa señala que en muchos casos la sola intervención del sistema de justicia penal no es suficiente para reparar el perjuicio y los daños ocasionados por el delito. Por eso, se considera la necesidad de crear otro tipo de intervención para auxiliar a las víctimas de conflictos penales, resolviendo sus pretensiones reales (Comité de Ministros del Consejo de Europa, 2014). 


\section{Revista Iberoamericana \\ de las Ciencias Sociales y Humanísticas}

ISSN: $2395-7972$

De ahí la necesidad de buscar alternativas al saturado y colapsado sistema de justicia formal, teniendo como la alternativa más viable el paradigma de la justicia alternativa y sus mecanismos alternos de solución de conflictos, los cuales encuentran cabida en el marco constitucional nacional en la reforma de junio de 2008, específicamente en el actual párrafo quinto del artículo 17 (Diario Oficial de la Federación [DOF], 18 de junio de 2008).

\section{Método}

Para el desarrollo del estudio y organización de los conocimientos se utilizaron los siguientes métodos:

Deductivo: Se partió de principios, modelos y paradigmas generales, como los modelos de mediación existentes y los paradigmas de administración de justicia con la finalidad de descender en realidades específicas, tal como el uso de un modelo en particular (como lo es el transformativo) y su desempeño en una problemática individualizada (como lo es la resolución de conflictos a través de la mediación en sede judicial).

Documental: La base principal de recopilación de información fueron los textos de carácter científicos (libros, artículos de revistas indexadas y normas jurídicas). Asimismo, se reunieron y compararon diversas ideologías y paradigmas según las obras utilizadas, por lo que se considera una investigación documental exploratoria.

Discursivo: Se abordó el objeto de estudio de forma indirecta, contemplándolo desde diversos puntos de vista, ópticas e ideologías para generar una idea precisa y completa, en este caso, el estudio de los modelos de mediación existentes en combinación con los sistemas de administración pública y los sistemas de resolución de conflictos.

Sistemático jurídico: Se reunieron elementos aparentemente aislados para convertirlos en una nueva realidad, unificando una nueva interpretación de estos, en este caso, el análisis de figuras autocompositivas de solución de conflictos y los paradigmas de administración pública. Se unificaron sus teorías para conocer el impacto que surte una en relación con la otra, específicamente el impacto que sufren los medios alternativos de solución de conflictos en relación con el cambio e imposición de nuevas ideologías de administración pública.

Funcionalista: Al analizar tanto el fenómeno de la mediación y las instituciones encargadas de impartirla en sede judicial, así como a la gerencia pública y las instituciones 


\section{Revista Iberoamericana \\ de las Ciencias Sociales y Humanísticas}

ISSN: 2395 - 7972

que hacen uso de tal ideología en relación con su capacidad de satisfacer necesidades sociales, específicamente en el rubro de administración de justicia y solución de conflictos.

\section{Resultados}

El fenómeno de la mediación cuenta con diversos modelos, los cuales nos ayudan a entender sus fines y objetivos; sin embargo, son tres los que se reconocen a nivel global como los más relevantes, por ser la base para el desarrollo del resto de modelos existentes dentro de la mediación; estos son el modelo de Harvard, el modelo circular narrativo y el modelo transformativo. Lo que se puede considerar como relevante dentro de este último es utilizar el conflicto como una oportunidad para transformar la conducta humana; es decir, se fomenta el crecimiento moral de cada persona a través de su revalorización (Baruch Bush y Ganong Pope, 2008). En este modelo, para solucionar un conflicto no se enfrenta un derecho personal con otro, sino que se busca el bien común y el comunitarismo. Por lo tanto, la prioridad es la satisfacción del interés general sobre el interés particular, reparando en lo posible el tejido social dañado por el conflicto (Márquez Algara, 2004) y planteando que la mediación es la oportunidad perfecta para que los individuos sean más solidarios entre sí (Izasa Gutierrez, 2018).

Sin embargo, también cuenta con detractores señalando como principal desventaja de la mediación transformativa que esta presenta un espacio que podríamos denominar de "desentendimiento" por parte del mediador, pues al dejar a los mediados que dirijan la comunicación dentro de la sesión, se convierte en un proceso de recriminaciones mutuas. Esta práctica de alejamiento por parte del mediador ha sido fomentada por el modelo transformativo al señalar que solo así se puede generar el fortalecimiento de las partes, que es piedra angular para la transformación de las visiones conductuales (Madrid Lira, 2016).

Antes de abordar de lleno el tema de la gerencia pública, es necesario especificar lo que se entiende por administración pública, definida en innumerables ocasiones por diversos tratadistas. De acuerdo con lo plasmado por Luther Gulick, es aquella parte de la administración que se entiende con el gobierno, "especialmente con la rama ejecutiva del mismo, que es la encargada de llevar a cabo el trabajo del gobierno, de tal modo la administración pública es una división de las ciencias políticas y una de las ciencias sociales” (Gómez Días de León, 2015, p. 24). 
Revista Iberoamericana

de las Ciencias Sociales y

Humanísticas

ISSN: $2395-7972$

Lamentablemente, el sistema de administración de justicia en el México contemporáneo se encuentra inmerso en una crisis de operatividad que trae aparejada como consecuencia un desgaste intenso en su credibilidad ante la sociedad. Esto se refleja dentro del Índice de Estado de Derecho 2017-2018, realizado por el World Justice Proyect, en donde México se ubicó en el lugar 99 de 126 países evaluados, índice que tasa temas como límite del poder gubernamental, ausencia de corrupción, gobierno abierto, derechos fundamentales, orden y seguridad, cumplimiento regulatorio, justicia civil y justicia penal.

Las puntuaciones obtenidas dentro de las diversas áreas en las que México fue evaluado son las siguientes:

a) límite del poder gubernamental 84/126;

b) ausencia de corrupción 117/126;

c) gobierno abierto $35 / 126$;

d) derechos fundamentales $73 / 126$;

e) orden y seguridad 117/126;

f) cumplimiento regulatorio $87 / 126$;

g) justicia civil 113/126 y,

h) justicia penal 115/126 (World Justice Project [WJP], 2018).

Una de las razones que han provocado la existencia de esta crisis se debe al aumento progresivo de los conflictos ante los tribunales, los cuales han generado una saturación y desestabilización del sistema jurisdiccional, ya que la cultura que nos han heredado se basa en la idea de que todo se debe resolver a través de violencia (esto es, mediante procedimientos jurisdiccionales). Como sociedad no buscamos realmente la justicia, sino que se castigue a la otra persona, el sufrimiento de la contraparte por cualquiera que haya sido su actuar, es decir, la venganza legalizada (García Carvajal, 2006). Debido a esto, se han adoptado paradigmas alternos de administración de justicia distintos a la ideología de la justicia retributiva, en la cual tienen especial relevancia las teorías encaminadas a la mínima intervención del Estado, tales como el abolicionismo penal, el minimalismo penal, el contractualismo de Nozick, el garantismo de Ferrajoli, el modelo consensual de Carlos Nino, el derecho penal de alternativas, la justicia restaurativa y la justicia alternativa, entre otras ideologías.

Claus Roxin considera que una de las tareas más importantes que tiene el derecho penal es la de encontrar soluciones a los conflictos que permitan restablecer la paz social, 


\section{Revista Iberoamericana de las Ciencias Sociales y Humanísticas}

toda vez que estos vienen a perturbar a la población. De igual forma, señala que esta siempre estará dispuesta a priorizar la conciliación y el diálogo para resolver sus conflictos, cuando se trate de delitos de poca monta o donde no se sufran daños físicos o materiales (Nino, 1980).

Es así como se transita el camino hacia la mínima intervención del Estado en la resolución de los conflictos sociales hasta llegar a la justicia restaurativa y su gran aliada, la justicia alternativa, dentro de la cual encuentran cabida los mecanismos alternos de solución de conflictos como medios eficaces de impacto en la disminución de la reincidencia del conflicto.

La implementación de los programas de mediación en sede judicial tiene como objetivo el acceso a la justicia, la derivación judicial, los descongestionamientos de los tribunales, la imagen institucional, la sensibilización de la mediación a la ciudadanía y a los funcionarios del Poder Judicial. El hecho de que la mediación y los sistemas de resolución sean dependientes de los poderes judiciales debe generar dos cambios importantes: primero, el cambio de la percepción de la ciudadanía que ahora debe conocer que se pueden tener soluciones armónicas a sus conflictos, y segundo, que el personal que administra justicia debe internalizar este hecho (Soler Mendizábal, 2011).

Ahora bien, a la par de los cambios suscitados en los paradigmas de la administración de justicia, el Estado mexicano opta por un cambio de ideología en la gestión pública y sus instituciones; esta nueva ideología es la denominada nueva gerencia pública (Sánchez González, 2010).

Dentro de las diversas definiciones con las cuales cuenta la NGP destacan dos de ellas: "se define como el conjunto de conocimientos y prácticas que permiten mejorar la racionalidad de la dirección administrativa del Estado en términos sociales" y "la gerencia pública supone un centrarse sobre una estrategia (más que sobre un proceso gerencial) sobre las relaciones interorganizacionales y sobre la intersección de la gerencia y la política públicas" (Cepeda Islas, 2006, p. 4).

La nueva gerencia pública es un intento por desprenderse de la burocracia weberiana, en donde el dirigente detentaba su posición de poder en un marco de competencias legales que lo ubicaban a la cabeza de un cuerpo administrativo rigurosamente jerarquizado y compuesto por individuos libres, los cuales eran sometidos a una vigilancia y disciplina rigurosa. Esta ideología, sin embargo, quedó desfasada después de la Segunda Guerra Mundial por sus bajos niveles de eficacia y altos pocentajes de discrecionalidad, opacidad y 
corrupción, lo cual abre las puertas a la gerencia pública (Martínez Vilchis, 2009). Como lo comenta Cepeda Islas, "la gerencia pública tiene entonces como objetivo el mejoramiento de la capacidad de gobierno para lograr un incremento de la gobernabilidad y tener en condiciones de mejora continua los procesos administrativos, y así resolver de manera eficiente y expedita los problemas que presenta la sociedad" (Cepeda Islas, 2006, p. 2).

No obstante, el choque de paradigmas que se advierte tiene que ver con los principios propios de la implementación de la nueva gerencia pública en la administración de justicia y específicamente en la justicia alternativa, en concordancia con los objetivos que se pretenden alcanzar con la aplicación del modelo transformativo de la mediación.

Los objetivos que persigue el modelo transformativo son la transformación de las ideas de las personas que intervienen en el proceso de mediación, generando una nueva visión de combate al conflicto, sobre todo de convivencia social y desarrollo personal; es decir, la culturización y moralización de las personas, mejorando las relaciones interpersonales entre los protagonistas del conflicto de forma profunda y permanente.

Lo antes plasmado cobra relevancia si se observan los pilares o características que dan sustento a la NGP, siendo estos:

a) el subíndice visión del ciudadano como cliente,

b) el subíndice mayor utilización de tecnología de información,

c) el subíndice descentralización,

d) el subíndice rendición de cuentas,

e) el subíndice planeación y gestión estratégica,

f) el subíndice contratos $y$,

g) el subíndice transparencia (Martínez Vilchis, 2009, p. 205).

Cobra especial relevancia el subíndice de "rendición de cuentas", el cual puede convertirse en una severa limitante para la mediación transformativa en sede judicial, pues — como ya se ha mencionado- consiste en la evaluación constante del desempeño administrativo sobre la utilización de los recursos públicos en función de las metas y objetivos planteados, siendo lo más importante el desempeño y resultados obtenidos. En este sentido, se prioriza la estadística y los números fríos por encima de los cambios sustanciales y beneficios de fondo que el servicio público pueda realizar; en este caso, la mediación en sede judicial a través del modelo transformativo. 


\section{Discusión}

Es necesario el análisis del fenómeno de la gerencia pública para que se establezca la viabilidad de su implementación y funcionabilidad dentro de las instituciones encargadas de la administración pública (en específico en la administración de justicia), pues su finalidad ulterior es transformar las instituciones gubernamentales para transitar del clásico aparato burocrático a un modelo empresarial, en donde el objetivo sea conseguir la satisfacción de las necesidades de las personas (Arellano y Cabrero, 2005).

La ideología de la nueva gerencia pública prioriza el aparato operativo, el formalismo y el número de personas atendidas por parte de las instituciones públicas, con lo que se deja de lado el efecto a mediano y largo plazo que puedan tener estos servicios. Tal situación obliga a los centros de mediación a generar convenios como línea de producción con la finalidad de justificar como adecuada su funcionalidad y, sobre todo, justificar el presupuesto otorgado anualmente.

A nivel nacional, el número de casos concluidos por los órganos, centros o unidades de justicia alternativa ha disminuido cerca de $30 \%$ entre los años 2013-2015, a pesar de que el personal que labora en los centros de justicia alternativa - considerando al personal operativo y administrativo a nivel nacional - ha aumentado en alrededor $20 \%$ en ese mismo periodo, mientras que el número de mediadores se ha mantenido (Martínez Solis, 2017).

Esta estructura de medición en donde se evaluarán a las instituciones o centros encargados de la justicia alternativa en sede judicial por el número de expedientes que resuelven sin importar el impacto social de ellos, o bien el tema de la reincidencia del conflicto, viene a destruir las esperanzas de una culturización y moralización por parte de la mediación, en específico de la mediación transformativa. Cabe destacar que los distintos modelos de mediación tienen como objetivo primordial el cambio estructural en las personas, lo cual necesita de tiempo suficiente para lograr estos cambios sustanciales en los mediados, tiempo que la nueva gerencia pública no otorgará, pues esto limitaría el número de casos o expedientes que se puedan resolver.

Lo anterior es así porque se obliga a los mediadores a preocuparse por lograr la firma de un acuerdo o convenio, lo que se traduce legalmente en la solución de un conflicto, aumentando de esta forma la estadística institucional, elemento esencial en la nueva gerencia pública, sin importar la moralización o cambio estructural de los mediados. 


\section{Conclusiones}

La reforma constitucional del 18 de junio de 2008 en materia penal —a través de la cual se instaura a la justicia alternativa como pilar del sistema penal acusatorio - trajo consigo una obligatoriedad para las entidades federativas de legislar y ofrecer dentro de sus marcos normativos locales la figura de los medios alternos de solución de conflictos y sus herramientas pacificadoras, siendo el propio Estado a través de sus centros de justicia alternativa dependientes del Poder Judicial los que tomaron la batuta para incursionar e implementar en México el paradigma restaurativo.

El ideal de la mediación en sede judicial es el debido funcionamiento y la actualización de los objetivos planteados por los modelos utilizados; sin embargo, el hecho de estar inmersos dentro del andamiaje estadual los hace víctimas dependientes de las deficiencias y carencias del propio Estado, como la falta de presupuesto, carencia de personal, insuficiencia en el material de trabajo y perpetuación de los vicios propios de las instituciones públicas. Esto limita en gran medida los alcances de la mediación y el tipo de modelo que puede ser utilizado, siendo el más popular por su practicidad ideológica el modelo de Harvard.

El modelo de mediación que debe de ser implementado en los centros de justicia alternativa en sede judicial es el transformativo, ya que tiene como objetivo principal la revalorización y moralización de los mediados, lo que impacta de forma directa en la disminución de los conflictos a través de la culturización, la prevención y la no reincidencia.

La adopción de la ideología de la gerencia pública es la respuesta que actualmente está permeando dentro de las instituciones o entes del Estado en México para dar respuesta a la crisis de justicia que prevalece en el estado mexicano, ideología de administración pública que pondera la eficiencia de las instituciones, adoptando las características, principios y fines a través de los cuales funcionan y se conciben las empresas privadas.

Lamentablemente, la implementación de la gerencia pública en la política de administración de justicia en México ha impactado de forma negativa en la aplicación de los mecanismos alternos de solución de conflictos, ya que es un modelo que se concentra en factores cuantitativos y estadísticos, los cuales miden su éxito según el número de asuntos que terminan con un convenio firmado, sin importar si este tiene un impacto sustancial o cualitativo en la visión y estructura del mediado. En otras palabras, lo importante es si ayuda a disminuir la reincidencia en el conflicto, situación que nos llevará inevitablemente a la 
saturación del sistema de justicia acusatorio, pues la justicia alternativa no es capaz de eliminar $90 \%$ de los asuntos totales que se presentan a instancias jurisdiccionales, lo que genera la ineficacia del sistema y su colapso. Como ejemplo de lo anterior, en el año 2017 ingresaron a los órganos, centros o unidades especializadas en el Sistema de Justicia Alternativa y/o Mecanismos Alternos de Solución de Controversias 158120 asuntos, según datos del Instituto Nacional de Estadística y Geografía.

Por todo ello, es indispensable eliminar la ideología de la gerencia pública en los centros de justicia alternativa en sede judicial, al limitar en gran medida las bondades y alcances que puede generar la mediación transformativa, y obligando al mismo tiempo a los operadores del sistema a utilizar solo un modelo de mediación (el modelo de Harvard), ya que es el que responde de mejor forma a las exigencias de la nueva gerencia pública.

\section{Referencias}

Arellano, D. y Cabrero, E. (2005). La nueva gestión pública y su teoría de la organización: ¿son argumentos antiliberales? Justicia y equidad en el debate organizacional público. Gestión y Política Pública, 14(3), 599-618.

Baruch Bush, R. A. y Ganong Pope, S. (2008). La mediación transformativa: un cambio en la calidad de la interacción en los conflictos familiares. Revista de Mediación, 1(2), 22.

Cejudo, G. (2011). Nueva gestión pública. México: Siglo Veintiuno.

Cepeda Islas, S. (2006). DOCPLAYER. Obtenido de DOCPLAYER.ES: https://docplayer.es/40293101-Que-es-la-gerencia-publica.html

Comisión Nacional de los Derechos Humanos [CNDH] (2018). Diagnóstico nacional de supervisión penitenciaria. Comision Nacional de los Derechos Humanos. México: CNDH México.

Comité de Ministros del Consejo de Europa (2014). Recomendación (87) 21, adoptada por el Comité de Ministros del Consejo de Europa el 17 de septiembre de 1987, sobre la asistencia a las víctimas y la prevención de la victimización. Recuperado de http://funvic.org/paginas/legislacion/legi5.htm

Diario Oficial de la Federación (18 de junio de 2008). Diario Oficial de la Federación. Recuperado de Secretaría de Gobernación: http://dof.gob.mx/nota_detalle.php?codigo=5046978\&fecha=18/06/2008 
Revista Iberoamericana

de las Ciencias Sociales y

Humanísticas

ISSN: $2395-7972$

Eiras Nordenstahl, U. C. (2010). Mediacion penal. De la práctica a la teoría. Buenos Aires, Argentina: Editorial Histórica.

Folger, J. (2006). Mediación transformativa: preservación del potencial propio de la mediación en escenarios de disputas. Revista de Medicación, 1(2), 36.

García Carvajal, E. C. (2006). Contexto Nacional de los Métodos Alternos de Solución de Conflictos. En Gorjón Gómez, F. J. Justicia aternativa médica. El derecho y la bioética en la ciudad internacional del conocimiento (pág. 154). Monterrey, Nuevo León, México: Universidad Autónoma de Nuevo Léon.

Giménez Romero, C. (2001). Modelos de mediación y su aplicación en mediación intercultural. Revista Migraciones, (10), 59-110.

Gómez Días de León, C. (2015). Origen y evolución del estudio de la administración pública. En Gómez Díaz de León, C. De la adminsitración pública tradicional a la nueva gestión pública. Evolución, conceptos y desafios (pp. 1-18). México: McGraw-Hill.

Guerrero, O. (2003). Nueva gerencia pública: ¿gobierno sin política? Revista Venezolana de Gerencia, 8(23), 379-395.

Hernández Ramos, C. (2014). Modelos aplicables en mediación intercultural. Barataria. Revista Castellano-Manchega de Ciencias sociales, (17), 67-80.

Izasa Gutierrez, J. P. (2018). Aplicación del modelo transformativo de mediación en la conciliación extrajudicial de Colombia. Revista de Paz y Conflictos, 11(1), 151.

León Corona, B. y Cruz Badillo, I. (2014). Evolución y desarrollo de la administración y gestión pública: un acercamiento a un estado del conocimiento en construcción, a través de la Revista Gestión y Política Pública. Edahi. Bolentín Científico de Ciencias Sociales y Humanidades, 2(4).

Madrid Lira, S. (2016). Mediación transformativa. Guía práctica. Recensión. Revista de Mediación, 9(2).

Márquez Algara, M. G. (2004). Mediación y administración de justicia. Hacia la consolidación de una justicia participativa. Aguascaliente, Aguascalientes, México: Universidad Autónoma de Aguascalientes.

Martínez Solis, D. (2017). La difusión de la mediación en el nuevo sistema de justicia penal acusatorio. Revista Iberoamericana de Producción Académica y Gestión Educativa, $4(8)$. 
Revista Iberoamericana

de las Ciencias Sociales y Humanísticas

ISSN: $2395-7972$

Martínez Vilchis, J. (2009). La nueva gerencia pública en México. Una medición de su intensidad e impactos en las entidades del país. Convergencia, 16(49).

Nató, A. M. (2006). Mediación comunitaria, conflcitos en el escenario social urbano. Buenos Aires, Argentina: Universidad .

Nino, C. S. (1980). Los límites de la responsabilidad penal, una teoría del delito. Buenos Aires: Astrea.

Pacheco Pulido, G. (2012). Mediación. Cultura de la paz. Medio alternativo de adminsitración de justicia. México: Porrúa.

Pineda Ortega, P. (2015). Génesis y caracterización de la nueva gestión pública en Latinoamérica. Contextualizaciones Latinoamericanas, 7(12).

Revilla González, J. A. (2007). La mediación penal. En Soleto Muñoz, H. Mediacion y solución de conflictos (pág. 302). España: Tecnos.

Salcedo Casallas, J. R. (2015). Nueva gestión pública en las “maneras de hacer” del docente universitario. Revista Educación y Desarrollo Social, 9(2), 102-123.

Sánchez González, J. J. (2010). ¿Innovando en la gestión pública? La experiencia mexicana en los gobiernos locales. Revista Espacios Públicos, 13, 23.

Soler Mendizábal, R. (2011). Mediación y conciliación en sede judicial. En Quiroga, M. (coord.), Métodos alternos de solución de conflictos. Herramientas de paz y modernización de la justicia (pp. 203-216). Madrid: Dykinson.

Villareal Solis, S. E. (2015). Administración pública: su transformación hacia la nueva gestión pública. En Gómez Díaz de León, C. De la administración pública tradicional a la nueva gestión pública Evolución, conceptos y desafios (pág. 138). Monterrey, Nuevo León, México: McGrawHill.

World Justice Proyect [WJP] (2018). Índice de Estado de Derecho 2017-2018. Washington D.C.: World Justice Project Rule of Law Index. 
Revista Iberoamericana

de las Ciencias Sociales y

Humanísticas

ISSN: $2395-7972$

\begin{tabular}{|l|l|}
\hline Rol de Contribución & Autor (es) \\
\hline Conceptualización & $\begin{array}{l}\text { José Antonio Serrano Morán(principal), Nicté Rosas } \\
\text { Topete(igual), Edgar Gabriel Ávila Verdín(igual) }\end{array}$ \\
\hline Metodología & $\begin{array}{l}\text { José Antonio Serrano Morán(igual), Nicté Rosas Topete(igual), } \\
\text { Edgar Gabriel Ávila Verdín(igual) }\end{array}$ \\
\hline Software & NO APLICA \\
\hline Validación & $\begin{array}{l}\text { José Antonio Serrano Morán(igual), Nicté Rosas Topete(igual), } \\
\text { Edgar Gabriel Âvila Verdín(igual) }\end{array}$ \\
\hline Análisis Formal & $\begin{array}{l}\text { José Antonio Serrano Morán(igual), Nicté Rosas Topete(igual), } \\
\text { Edgar Gabriel Ávila Verdín(igual) }\end{array}$ \\
\hline Investigación & $\begin{array}{l}\text { José Antonio Serrano Morán(principal), Nicté Rosas } \\
\text { Topete(igual), Edgar Gabriel Ávila Verdín(igual) }\end{array}$ \\
\hline Recursos & $\begin{array}{l}\text { José Antonio Serrano Morán(igual), Nicté Rosas Topete(igual), } \\
\text { Edgar Gabriel Ávila Verdín(igual) }\end{array}$ \\
\hline Curación de datos & NO APLICA \\
\hline $\begin{array}{l}\text { Escritura - Preparación del } \\
\text { borrador original }\end{array}$ & $\begin{array}{l}\text { José Antonio Serrano Morán(igual), Nicté Rosas Topete(igual), } \\
\text { Edgar Gabriel Ávila Verdín(igual) }\end{array}$ \\
\hline $\begin{array}{l}\text { Escritura - Revisión y } \\
\text { edición }\end{array}$ & $\begin{array}{l}\text { José Antonio Serrano Morán(igual), Nicté Rosas Topete(igual), } \\
\text { Edgar Gabriel Ávila Verdín(igual) }\end{array}$ \\
\hline Visualización & $\begin{array}{l}\text { José Antonio Serrano Morán(igual), Nicté Rosas Topete(igual), } \\
\text { Edgar Gabriel Âvila Verdín(igual) }\end{array}$ \\
\hline Supervisión & $\begin{array}{l}\text { José Antonio Serrano Morán(principal), Nicté Rosas } \\
\text { Topete(igual), Edgar Gabriel Âvila Verdín(igual) }\end{array}$ \\
\hline $\begin{array}{l}\text { Administración de } \\
\text { Proyectos }\end{array}$ & $\begin{array}{l}\text { José Antonio Serrano Morán(principal), Nicté Rosas } \\
\text { Topete(igual), Edgar Gabriel Ávila Verdín(igual) }\end{array}$ \\
\hline Adquisición de fondos & $\begin{array}{l}\text { José Antonio Serrano Morán(principal), Nicté Rosas } \\
\text { Topete(igual), Edgar Gabriel Ávila Verdín(igual) }\end{array}$ \\
\hline
\end{tabular}

